\title{
An Unusual Case of Frequent Knee Locking
}

\author{
Chandrakant Nayak ${ }^{1}$, Baroda Prasanna Samal ${ }^{1}$, Mantu Jain ${ }^{1 *}$ and Bhaskar Rao ${ }^{2}$ \\ ${ }^{1}$ Department of Orthopedics, AIIMS, India \\ ${ }^{2}$ Department of Anesthesia, AIIMS, India
}

*Corresponding author: Dr Mantu Jain, Department of Orthopedics, AIIMS, 106 mahedev Orchid, Cosmoplois Road, Dumduma, Bhubaneswar Odisha, India

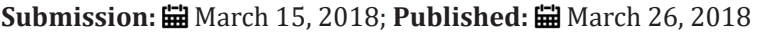

\begin{abstract}
Frequent knee locking is an incapacitating condition that requires a thorough clinical, radiological and arthroscopic evaluation. The common causes are meniscal injury followed by torn anterior cruciate ligament and osteochondral loose bodies in the joint space. Our case describes an unusual case of frequent left knee locking. After clinical and radiological examinations the provisional diagnoses of medial meniscus tear was done. A diagnostic arthroscopy of the left knee revealed normal meniscus, cruciates and a medial patello femoral plica slipping into the patellofemoral joint causing the locking sensation. The plica was resected. At 6 month follow up there is no recurrence of symptoms.
\end{abstract}

Keywords: Knee locking; Meniscus; Medial plica; Resection

\section{Introduction}

An alar fold exists between the patellofemoral and tibio femoral joint space both medially and laterally. Hypertrophic alar folds are called plicas. Morphologically there are infrapatellar, suprapatellar, mediopatellar and lateral patellar types of plicas [1], but medial plicas most commonly causes symptoms. Plicas slipping into patellofemoral joints and causing locking symptoms are very scantily reported in the literature. Here we present a case of medial plica causing frequent knee locking sensations and relevant clinical significance.

\section{Case Report}

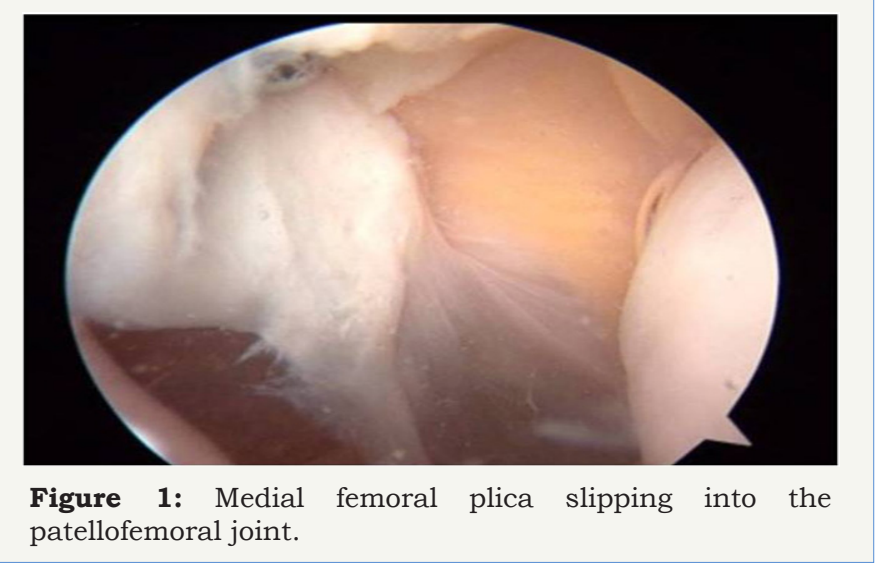

35 year old lady presented with one year history of severe occasional pain and locking sensation. She had no definite history of trauma or twisting injury. Physical examination revealed mild swelling on her left knee and medial joint line tenderness. Range of motion was not limited. Clinical examination for cruciates and meniscuses came out to be normal. Radiograph was normal. MRI showed degenerative changes over menisci but no definite tear. Diagnostic arthroscopy was planned after 6 months of conservative treatment and follow up. Both the menisci and cruciates were normal. Thickened medial plica mimicking a fibrotic band was seen on medial aspect of knee impinging on medial edge of medial femoral condyle (Figure 1). Plica was resected (Figure 2). ICRS grade 2 changes over cartilage on medial femoral condyle seen. Cartilage injury was grade 2 according to Outer bridge classification (Figure 3). Patient was referred to our physiotherapy unit for her quadriceps strengthening. Post operatively the patient experienced complete resolution and no recurrence of symptoms for last 6 months.

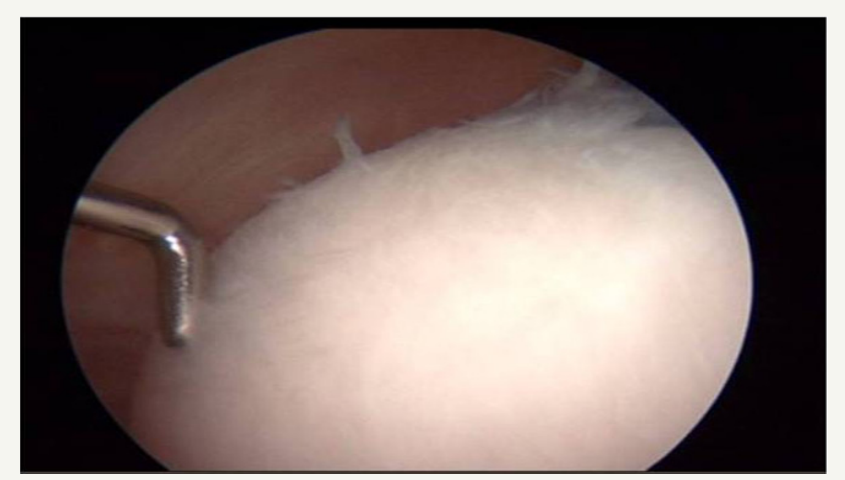

Figure 2: ICRS grade II changes in femoral cartilage. 


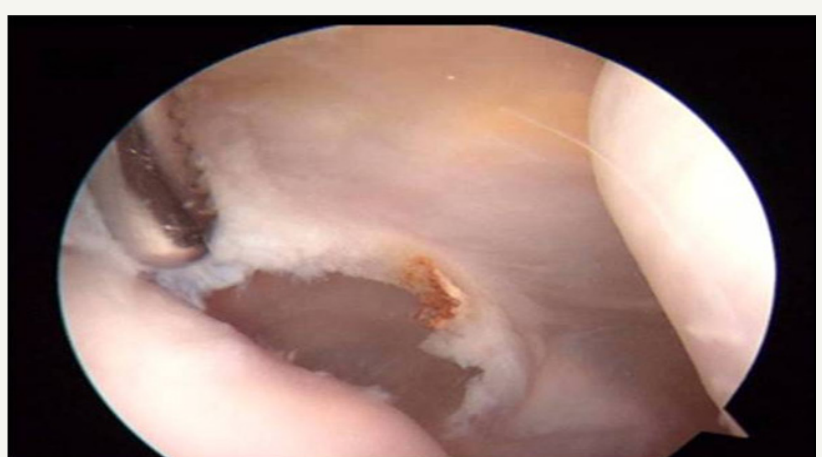

Figure 3: Plica being resected.

Discussion

First described by Lino in 1939 synovial plica of the knee is identified as a normal structure that represents traces of the synovial membrane in embryological development of the knee [2]. According to its corresponding anatomical site of origin, four types of synovial plicae can be identified in relationship to the patella as supra patellar, infra patellar, medial and lateral patellar plicae [3]. The normal plica is a thin and flexible fold containing elastic connective tissue. The elasticity of the tissue allows the plica to slide over bone prominence. Trauma to the knee can cause inflammation, resulting in swelling, thickening and decreased elasticity of the tissue. The plicae may eventually scar into fibrotic bands that "bowstring" across the femoral condyle and then it is responsible for the so-called plica syndrome [4]. This synovial plica is not present in all individuals. The average incidence of the plica in an adult population is stipulated between $20 \%$ and $60 \%$ [5]. In arthroscopic procedures, the frequency with which the presence of the synovial plica has been reported varies among 14\% and $100 \%$, and among $25 \%$ and $33 \%$ in cadaver dissections [6]. Supra patellar is among the most common and lateral patellar plica is the least common. The mediopatellar plica is the most common one attributed to the development of the plica syndrome. This plica is found in the coronal plane; it originates in the supra-patellar region and passes inferiorly on the medial aspect of the knee joint before inserting into the infra-patellar (Hoffa) fat pad. Based on its appearance according to Sakakibara, it is classified as type A (cord-like), type B (shelf-like, but not covering the medial femoral condyle), type $\mathrm{C}$ (covering the medial femoral condyle), and type D (having a double insertion) [1]. The most common symptoms associated to synovial plica include pain in the anterior part of the knee, as well as, edema associated with clicking, infection, blocking or pseudo blocking of the knee [7]. It is thought that the symptoms are due to the impingement of plica by the femoral condyle during the flexion [8]. Ours was a medial plica of type C. A medial plica causing frequent locking and occasional pain mimicking meniscus tear is not well described in literature. The treatment of synovial plica initially must be conservative with changes of daily physical activities, stretching exercises of anterior and posterior quadriceps muscles, occasional knee immobilization in the extended position for a few days associated with the use of anti-inflammatory, if the patient presents no improvement of the symptoms, the indication of arthroscopic removal of this plica should be evaluated [9].

\section{Conclusion}

The plica syndrome has been well described but there frequent knee locking because of plica is not much described in literature. So our report illustrates the importance of maintaining high index of suspension for the diagnosis of medial plica. It also suggests the fact that in presence of a normal radiological picture, locking of knee can be due to medial plica which should be kept in mind in differential diagnoses of knee locking.

\section{References}

1. Dupont J (1977) Synovial plicae of the knee. Controversies and Review. Clin Sport Med 16(1): 87-122.

2. Tindel N, Nisonson B (1992) The plica syndrome. Orthop Clin North Am 23(4): 613-618.

3. Hardaker WT, Whipple TL, Bassett FH (1980) Diagnosis and treatment of the plica syndrome of the knee. J Bone Joint Surg Am 62(2): 221-225.

4. Cothran RL, McGuire PM, Helms CA, Major NM, Attarian DE (2003) MR imaging of infrapatellar plica injury. Am J Roentgenol 180(5): 14431447.

5. Kinnard P, Levesque R (1984) The plica syndrome. A syndrome of controversy. Clin Orthop Relat Res 183: 141-143.

6. Klein W (1983) The medial shelf of the knee. A follow-up study. Arch Orthop Trauma Surg 102(2): 67-72.

7. Johnson P, Eastwood M, Witherow J (1993) Symptomatic synovial plicae of the knee. J Bone Surg Ser 75(10): 1485-1496.

8. Jackson R, Marshall D, Fujisawa Y (1982) The pathologic medical shelf. Orthop Clin North Am 13(2): 307-312.

9. Maffulli N, Testa V, Capasso G (1993) Mediopatellar synovial plica of the knee in athletes: results of arthroscopic treatment. Med Sci Sport Exerc 25(9): 985-988.
Creative Commons Attribution 4.0 International License

For possible submissions Click Here

\section{Submit Article}

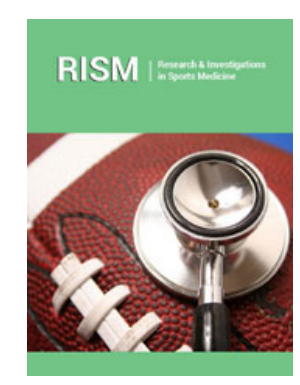

Research \& Investigations in Sports Medicine

\section{Benefits of Publishing with us}

- High-level peer review and editorial services

- Freely accessible online immediately upon publication

- Authors retain the copyright to their work

- Licensing it under a Creative Commons license

- Visibility through different online platforms 